\title{
Bioética e investigación: análisis de caso de la epidemia por el virus Ébola en 2014
}

\section{Bioethics and research: case study analysis of Ebola virus epidemic in 2014}

\section{Bioética e investigação: análise de caso epidemia do vírus ebola em 2014}

Fecha de recepción: 27 de junio de 2016

Fecha de evaluación: 9 de septiembre de 2016

Fecha de aceptación: 15 de noviembre de 2016

Disponible en línea: 16 de noviembre de 2016

\author{
Boris Julián Pinto Bustamante* \\ Ana Isabel Gómez Córdoba** \\ Luz Maira Wintaco Martínez ${ }^{* * *}$ \\ Diana Patricia Amador Muñoz $z^{* * * *}$ \\ Germán Andrés González Melo**** \\ Mary Lilián Carabalí Isajar ${ }^{* * * * * *}$ \\ Mónica Ararat Sarria ${ }^{* * * * * *}$ \\ Nury Nathalia Olaya Galán" \\ Luz Helena Patiño Blanco \\ Alfredo José Sanabria Solano"
}

Médico cirujano, especialista y magister en bioética Universidad El Bosque, candidato a doctorado en bioética Universidad El Bosque. Profesor investigador Bioética Universidad El Bosque. Profesor principal de carrera, Escuela de Medicina y Ciencias de la Salud, Universidad del Rosario. Domicilio: Carrera 69 D \# 96-39 Interior 1 apartamento 502. Teléfono: (571) 3021886. Correo electrónico: boris.pinto@urosario.edu.co. ORCID http://orcid.org/0000-0002-2463-4214. Bogotá, Colombia.

** $\quad$ Médica Pediatra, especialista en gerencia en salud pública y derecho médico sanitario de la Universidad del Rosario, magister en bioética de la Universidad del Bosque, doctora en Ciencias Jurídicas Pontificia Universidad Javeriana. Profesora titular, vice-decana Escuela de Medicina y Ciencias de la Salud Universidad del Rosario y co-directora de la especialización de derecho médico sanitario de la Universidad del Rosario. Domicilio: Calle 105 número 47-03. Teléfono: (571) 2570079. Correo electrónico: anai.gomez@urosario.edu.co. ORCID: http://orcid.org/00000001-6328-917X. Bogotá, Colombia. 
Dor: http://dx.doi.org/10.18359/rlbi.1943

\section{Cómo citar:}

Pinto Bustamante, B. J., Gómez Córdoba, A. I., Wintaco Martínez, L. M., Amador Muñoz, D. P. et al. (2016). Bioética e investigación: análisis de caso de la epidemia por el virus Ébola en 2014. Revista Latinoamericana de Bioética, 17(1), 124-149. Dor: http://dx.doi.org/10.18359/rlbi.1943

*** Bacterióloga y laboratorista clínico. Maestría en Ciencias Básicas Biomédicas-Universidad del Bosque. Estudiante de Doctorado en Ciencias Biomédicas y Biológicas. Escuela de Medicina y Ciencias de la Salud - Facultad de Ciencias Naturales y Matemáticas. Universidad del Rosario. Afiliación institucional: Grupo de Investigaciones Microbiológicas Universidad del Rosario (GIMUR).Correo electrónico: luz.wintaco@urosario.edu.co. ORCID: http://orcid.org/00000002-0126-0221. Bogotá, Colombia.

**** Médica cirujana, Magister Ciencias Biomédicas. Profesora de carrera, departamento de fisiología, Universidad del Rosario. Estudiante de Doctorado en Ciencias Biomédicas y Biológicas. Escuela de Medicina y Ciencias de la Salud - Facultad de Ciencias Naturales y Matemáticas. Universidad del Rosario. Correo electrónico: diana.amador@urosario.edu.co. ORCID: http://orcid.org/00000001-9984-4550. Bogotá, Colombia.

$* * * *$ Ingeniero Forestal. Estudiante de Doctorado en Ciencias Biomédicas y Biológicas. Escuela de Medicina y Ciencias de la Salud - Facultad de Ciencias Naturales y Matemáticas. Universidad del Rosario.Correo electrónico: germana.gonzalez@urosario.edu.co. ORCID: http://orcid.org/00000003-0069-456X. Bogotá, Colombia.

****** Bióloga, Magíster en Microbiología. Estudiante de Doctorado en Ciencias Biomédicas y Biológicas. Escuela de Medicina y Ciencias de la Salud - Facultad de Ciencias Naturales y Matemáticas. Universidad del Rosario. Afiliación institucional: Fundación Instituto de Inmunología de Colombia (FIDIC). Correo electrónico: mary_carabali@fidic.org.co. ORCID: http://orcid.org/0000-00026012-2101. Bogotá, Colombia.

${ }^{* * * * * *}$ Médico Cirujano, Magíster en Genética Humana. Estudiante de Doctorado en Ciencias Biomédicas y Biológicas. Escuela de Medicina y Ciencias de la Salud - Facultad de Ciencias Naturales y Matemáticas. Universidad del Rosario. Afiliación institucional: Fundación Instituto de Inmunología de Colombia (FIDIC). Correo electrónico: monica.ararat@urosario.edu.co. ORCID.org/0000-0002-5273-3260. Bogotá, Colombia.

${ }^{* * * * * * *}$ Microbióloga, Magister Ciencias Biológicas. Estudiante de Doctorado en Ciencias Biomédicas y Biológicas. Escuela de Medicina y Ciencias de la Salud - Facultad de Ciencias Naturales y Matemáticas. Universidad del Rosario. Afiliación institucional: Grupo de Enfermedades Infecciosas, Dpto. de Microbiología, Pontificia Universidad Javeriana. Correo electrónico: nury.olaya@urosario.edu.co. ORCID: http://orcid.org/0000-0002-4282-4951. Bogotá, Colombia.

Bacterióloga, Magister Microbiología. Estudiante de Doctorado en Ciencias Biomédicas y Biológicas. Escuela de Medicina y Ciencias de la Salud - Facultad de Ciencias Naturales y Matemáticas. Universidad del Rosario.Correo electrónico: luzh.patino@urosario.edu.co. ORCID: http://orcid.org/0000-0002-8667-8896. Bogotá, Colombia.

********* Ingeniero Biomédico. Estudiante de Doctorado en Ciencias Biomédicas y Biológicas. Escuela de Medicina y Ciencias de la Salud - Facultad de Ciencias Naturales y Matemáticas. Universidad del Rosario. Correo electrónico: alfredo.sanabria@urosario.edu.co.

ORCID: http://orcid.org/0000-0001-6803-6083. Bogotá, Colombia. 
Boris J. Pinto B., Ana I. Gómez C., Luz M. Wintaco M., Diana P. Amador M., Germán A. González M., Mary L. Carabalí I., Mónica Ararat S., Nury N. Olaya G., Luz H. Patiño B., Alfredo J. Sanabria S.

\title{
Resumen
}

La epidemia por el virus Ébola, en África occidental (2014), ha suscitado una serie de interrogantes éticos en torno a las medidas de salud pública para su contención, el uso de medicamentos experimentales y el desarrollo de vacunas contra esta enfermedad. El presente trabajo explora algunas de estas preguntas desde la perspectiva de la ética en investigación biomédica. La epidemia por el virus Ébola es un modelo de estudio adecuado para abordar esfuerzos multilaterales en investigación, así como para analizar aspectos antropológicos en salud pública y determinantes sociales, económicos y políticos en salud a nivel global.

Palabras clave: bioética, Ébola, enfermedad, ética en investigación, vacunas, virus.

\begin{abstract}
The Ebola virus epidemic in West Africa (2014) has raised some ethical questions surrounding public health measures for its containment, the use of experimental drugs and the development of vaccines against this disease. This paper explores some of these issues from the perspective of ethics in biomedical research. Ebola virus epidemic is a suitable study model to address multilateral efforts in research as well as to analyze anthropological aspects of public health and social, economic and political determinants of global health.
\end{abstract}

Keywords: Bioethics, Ebola, research ethics, vaccines, virus.

\section{Resumo}

A epidemia pelo vírus Ebola, na África Ocidental (2014), tem levantado uma série de questões éticas em torno às medidas de saúde pública para a sua contenção, o uso de medicamentos experimentais e o desenvolvimento de vacinas contra esta doença. Este artigo explora algumas destas questões a partir da perspectiva da ética na pesquisa biomédica. A epidemia pelo vírus Ebola é um modelo de estudo adequado para abordar esforços multilaterais em pesquisa, como também para analisar aspectos antropológicos em saúde pública e determinantes sociais, econômicos e políticos em saúde a nível global.

Palavras-chave: doença pelo vírus Ebola, bioética, ética em investigação, vacinas contra o Ebola. 


\section{Introducción}

La epidemia por el virus del Ébola (VE), en África Occidental en 2014, ofrece múltiples puntos de reflexión moral que sobrepasan los problemas de la microbioética, relacionados con la atención de los pacientes o la investigación con sujetos humanos. Esta epidemia es el indicio de una crisis global, el efecto de la profunda inequidad que caracteriza a la humanidad en este momento histórico y que encuentra un escenario ideal en África Occidental (Benatar, 2015).

El caso índice parece corresponder a un niño de dos años, habitante de Meliandou, una pequeña aldea cercana a Guéckédou en la República de Guinea (Marí Sáez et al., 2015). Los primeros casos se reportaron en Guinea desde marzo de 2014 y la epidemia se extendió hasta Sierra Leona, Liberia, Nigeria, Senegal, Mali, España y Estados Unidos por medio de viajeros que se movilizaban por vía terrestre o aérea (Organización Mundial de la Salud [oms], 2015a). La propagación mediante transmisión persona-persona, desde Guinea hasta Sierra Leona, ha sido ratificada en estudios de genómica (Yozwiak, Schaffner y Sabeti, 2015).

El 8 agosto de 2014, la oms, tras el reporte de 1779 casos y 961 muertes en Guinea, Sierra Leona, Nigeria y Liberia, declaró la epidemia por ve en África Occidental como una "emergencia de salud pública de preocupación internacional" (Gostin, Lucey y Phelan, 2014). En septiembre de 2014 se habían reportado 4507 casos probables y comprobados, así como 2296 muertes (oms, 2014a). En noviembre, la cifra ascendió a 13042 casos reportados y 4818 muertes (Lyon et al., 2014). El 15 de agosto de 2015 se reportaban 2127 casos y 1145 muertes en la zona de Guinea, Sierra Leona, Liberia y Nigeria. Para noviembre de 2015 , la oms reportó 28637 casos y 11315 muertes (oms, 2015b); la epidemia parece ingresar en una curva descendente desde la primera mitad de año 2015. La epidemia de 2014 es considerada la más crítica debido al número de víctimas, la rápida propagación, la extrema pobreza de los países afectados y la fragilidad de sus sistemas de salud (Donovan, 2014).

La transmisión de la enfermedad a un limitado número de personas provenientes de países industrializados incrementó las alarmas: un médico americano, una misionera americana y un religioso español (Lyon et al., 2014), los cuales fueron tratados con un medicamento experimental que se encontraba en fase I de desarrollo y que solo había sido probado en primates macacos (Macaca mulatta) (Lyon et al., 2014).

Esta situación generó una controversia mundial sobre las consideraciones biopolíticas de la enfermedad por VE, sobre aspectos éticos frente al uso de medicamentos experimentales y el desarrollo de vacunas en este contexto (Griffiths, 2014) y sobre el súbito interés en el ámbito internacional, particularmente al ser considerada esta enfermedad como un asunto prioritario en relación con la seguridad nacional en Estados 
Boris J. Pinto B., Ana I. Gómez C., Luz M. Wintaco M., Diana P. Amador M., Germán A. González M., Mary L. Carabalí I., Mónica Ararat S., Nury N. Olaya G., Luz H. Patiño B., Alfredo J. Sanabria S.

Unidos (Rid y Emanuel, 2014a). En 2014, el Centro para la Prevención y el Control de Enfermedades (Centers for Disease Control and Prevention [CDC]) propone la restricción de viajes locales e internacionales con el propósito de preservar la seguridad nacional, los intereses económicos y la salud pública de países ajenos a la epidemia (CDC, 2015a; Bogoch et al., 2015).

En vista de que en el momento del brote no se disponía de un tratamiento efectivo, el 11 de agosto de 2014 la oms propuso una mesa de discusión para analizar las consideraciones éticas relativas al uso de intervenciones no comprobadas en humanos, en el contexto de la epidemia por VE (oms, 2014b). Como lo señala la Comisión Presidencial para el Estudio de Problemas Bioéticos de los Estados Unidos (2015), las emergencias que afectan la salud pública, a las que se suman el miedo y la sensación de urgencia, son un reto para la interpretación de los principios éticos que guían la investigación con sujetos humanos.

El propósito de este artículo de revisión es identificar algunos aspectos bioéticos implícitos en esta epidemia, como modelo de análisis de la ética en investigación, a partir de las siguientes preguntas orientadoras: ¿Es ética la utilización de medicamentos no autorizados, experimentales, en el contexto de la epidemia por VE?, ¿quiénes deben ser los primeros en recibir los medicamentos, de los cuales hay pocos suministros? El dinero que se invierte en el desarrollo de estos fármacos, ¿estaría mejor invertido en suministros para la cuarentena y educación pública con el objetivo de ayudar a prevenir la transmisión de la enfermedad? El tratamiento con medicamentos experimentales contra el VE, ¿debería ofrecerse exclusivamente en el contexto de ensayos clínicos controlados aleatorizados?, ¿o existen otras alternativas que permitan generar conocimiento sobre el perfil de seguridad y eficacia de tales medicamentos?, ¿qué consideraciones éticas se deben tomar en cuenta en el desarrollo de vacunas contra el VE?, ¿cómo deben recolectarse, almacenarse y compartirse los materiales biológicos para investigación?

\section{La epidemia por el virus del Ébola en África Occidental (2014)}

Los primeros casos de enfermedad por el VE se reportaron en África central durante el primer brote simultáneo en la República Democrática del Congo (Yambuku) y en Sudán (Nzara) en 1976. La enfermedad por el ve en humanos es una condición hemorrágica febril de rápida evolución (Pourrut et al., 2005), con un periodo de incubación de 3 a 21 días, caracterizada por hemorragia, falla multiórganica y shock (Bausch, Sprecher, Jeffs y Boumandouki, 2008). Feldmann, et al (1993) reportan cuatro serotipos de la familia Filoviridae (filovirus): E. sudan, E. zaire, E. ivory coast y E. reston. Por su parte, la oms (2014) reporta dos serotipos adicionales: E. bundibugyo y $E$. tai forest. Los brotes que se han docu- 
mentado hasta la fecha solo involucran a E. bundibugyo, E. zaire, E. sudan. En la epidemia de 2014 se ha identificado la presencia de la especie zaire (Pourrut et al., 2005; oms, 2015a).

De acuerdo con la oms (2015a), las tasas de mortalidad de la enfermedad por VE han alcanzado valores entre el $25 \%$ y $90 \%$ durante los brotes previos a la actual epidemia. Las tasas de letalidad varían según el serotipo implicado: 88 \% para E. zaire (Marí Sáez et al., 2015) y $50 \%$ para E. sudan (Smith, 1978). Los estudios sobre distribución natural de este virus afirman una mayor concentración en África ecuatorial (Pourrut et al., 2005), particularmente en aldeas remotas y cercanas a la selva tropical (oms, 2015a).

Desde 1976 hasta 2014 se habían producido 25 brotes de enfermedad por VE, principalmente en África Central y Occidental, en países como República Democrática del Congo, Sudán, Gabón, Costa de Marfil, Sudáfrica y Uganda. Los brotes en las aldeas han sido precedidos, generalmente, por una epidemia en especies susceptibles a la infección por el VE (grandes simios, pequeños antílopes africanos) (Marí Saéz et al., 2015). En la epidemia de 2014 no se evidenció una merma en la población de primates no humanos en el sitio de origen de la epidemia (Guinea). Por su parte, la exposición masiva a grandes colonias de murciélagos insectívoros parece ser el origen de este brote epidémico, a través de un único evento de transmisión zoonótica (el contacto entre un niño de dos años y una colonia de murciélagos insectívoros de cola libre en un árbol hueco), seguida de la transmisión persona-persona, en una región caracterizada por la intensa modificación ecológica favorecida por actividades productivas humanas. La epidemia de 2014 representa la primera evidencia de la presencia del serotipo zaire en África Occidental (Marí Saéz et al., 2015).

Según cifras oficiales, entre 1976 y 2012 murieron 1590 personas, de 2387 infectadas, en la República Democrática del Congo, Uganda, Sudán, Gabón, Sudáfrica y Costa de Marfil, con un porcentaje de letalidad de casi el 67\% (oms, 2015a). Se han reportado brotes que han dejado una mortalidad superior a 200 personas en tan solo un año (mortalidad de 431 en Sudán y República Democrática del Congo en 1976; mortalidad de 254 en la ciudad de Kikwit en 1995) (Pourrut et al. 2005). Durante el brote de 2014, los países con mayor número de personas afectadas fueron Guinea y Sierra Leona, con tasas respectivas de mortalidad del $41 \%$ y $62 \%$ de los casos confirmados y $72 \%$ y $100 \%$ de los casos probables (oms, 2015c). Los tres países más afectados son algunos con menor inversión pública en salud del continente africano (Farmer, 2014).

La enfermedad por el ve es el resultado de un proceso de transferencia zoonótica (al igual que el paso del virus del viH al ser humano). Los primates y pequeños antílopes africanos son huéspedes susceptibles, mientras que algunos 
Boris J. Pinto B., Ana I. Gómez C., Luz M. Wintaco M., Diana P. Amador M., Germán A. González M.,

Mary L. Carabalí I., Mónica Ararat S., Nury N. Olaya G., Luz H. Patiño B., Alfredo J. Sanabria S.

murciélagos frugívoros (Epomophorus wahlberg) e insectívoros (Chaerephon pumilus y Mops condylurus) parecen ser los reservorios del virus (Marí Sáez et al., 2015). La infección directa a los humanos puede explicarse por la cacería de estos murciélagos para el consumo de su carne (Mickleburgh, Waylen y Racey, 2009). Hasta el momento no se ha demostrado transmisión aérea del vE. Inicialmente, su forma de transmisión es dada por medio del contacto de humanos con secreciones y líquidos de animales infectados (primates, antílopes de bosque, cerdos salvajes y puercoespines). Una vez ingresa a la comunidad se disemina por contacto directo con sangre y secreciones corporales de personas infectadas (Pourrut et al., 2005; CDC, 2015a), así como por transmisión sexual (CDC, 2015a).

La enfermedad por ve se inscribe dentro de los círculos epidémicos que trazan la extrema pobreza, prácticas culturales inseguras, las guerras civiles (Pérez y Faccini-Martínez, 2015), la corrupción institucional y la persistencia de prácticas coloniales en el continente africano. Se estima que el $62 \%$ de las muertes en esta región se derivan de enfermedades infecciosas y parasitarias (Mathers et al., 2009), dentro de las que se encuentran la malaria y las infecciones respiratorias (Mathers y Loncar, 2006; Mathers et al., 2009; Murray et al., 2012). Con el $11 \%$ de la población mundial, África aporta el $66 \%$ de los nuevos casos de infección por viH que se reportan cada día (Programa Conjunto de las Naciones
Unidas sobre el vir/Sida [Onusida], 2015), la cual es responsable del 35\% de las muertes en el continente africano (Mathers et al., 2009).

El brote del ve 2013-2014 impactó negativamente el comercio, provocó cierre de fronteras, suspensión de servicios de aerolíneas, cierre de escuelas, de hospitales, cortes en las cadenas de suministros, escasez de alimentos y de combustibles, disminución del turismo y de la inversión extranjera en la región de África Occidental, con una reducción significativa de su producto interno bruto (PIB) y los ingresos per cápita (United Nations Development Group [UNDG], 2015; Farmer, 2014).

\section{Cuestionamientos}

\section{¿Es ética la utilización de medicamen- tos no autorizados, experimentales, en el contexto de la epidemia por el virus de Ébola?}

Actualmente no existe reporte de un tratamiento específico para la enfermedad por el ve. Las opciones terapéuticas consisten principalmente en medidas de soporte, transfusión de productos sanguíneos, rehidratación, administración de antivirales y métodos de barrera para prevenir la trasmisión. Recientemente, se ha contemplado un rango de productos experimentales: proteínas recombinantes, ácido ribonucleico (ARN) de interferencia, anticuerpos monoclonales, bloqueadores de canales de iones (amiodarona, dronedarona, verapamilo) 
(Gehring et al., 2014), entre otros (Kilgore, Grabenstein, Salim, Rybak, 2015). Solo las intervenciones experimentales basadas en anticuerpos han demostrado beneficios sustanciales en modelos de primates cuando se administran 24 horas después de la exposición al virus (Qiu et al., 2014).

En 2012 se produjo ZMab, un anticuerpo monoclonal murino que, combinado con adyuvantes basados en adenovirus, proveía protección completa en macacos rhesus cuando se administraba 72 horas posterior a la infección. El anticuerpo fue humanizado y optimizada su forma de presentación, y se obtuvo como producto final un coctel de tres anticuerpos monoclonales altamente purificados, producidos transgénicamente en plantas de tabaco bajo la marca registrada ZMapp, de la casa farmacéutica MappBio. Dicha marca fue probada en 2014, sin haber sido sometida a ensayos clínicos, en dos trabajadores de la salud norteamericanos infectados, que se encontraban hospitalizados en Liberia; con esto se obtuvieron resultados positivos en cuanto a supervivencia (Qiu, et al., 2014; Mapp Biopharmaceutical, 2014; Kroll, 2014).

El medicamento se encuentra en una fase inicial de desarrollo, pero para el tiempo de utilización se desconocían evidencias sobre los efectos que se podrían derivar de su empleo (Lyon et al., 2014). En diciembre de 2014, se conocieron los resultados clínicos de dos pacientes en Estados Unidos infectados con el VE y tratados con el suero experimental
ZMapp. Sin embargo, de acuerdo con las recomendaciones del estudio, no se debe extrapolar el éxito de estos resultados en toda una población de forma prematura (Lyon et al., 2014).

En la reunión de la oms (2014b) del 11 de agosto de 2014, constituida por un panel de doce miembros seleccionados por su experiencia y representación geográfica, tras una discusión de tres horas por teleconferencia, se concluyó por unanimidad que es aceptable éticamente el uso de tratamientos no comprobados, con resultados promisorios en modelos animales sin evaluación previa en humanos, con fines potencialmente terapéuticos o preventivos. A este pronunciamiento se han sumado otras voces en favor del uso de medicamentos no autorizados en pacientes afectados por el ve (Frieden, 2014; Gostin et al., 2014; McCarthy, 2014).

Si bien esta decisión ha sido ampliamente respaldada, también se registran posiciones críticas: la administración de un tratamiento médico sin probar, y sin controles, es inaceptable para algunos, pues expone a una población vulnerable, susceptible de ser coaccionada ante falsas expectativas, a un riesgo desproporcionado (Griffiths, 2014). Algunos autores recurren al principio de precaución e indican que no deberían suministrarse medicamentos sin evidencia disponible de su perfil de riesgo y eficacia en humanos (Shah et al. 2015). Otros consideran que tienen mayor impacto las investigaciones que busquen optimizar la terapia con líquidos y electrolitos para evitar 
Boris J. Pinto B., Ana I. Gómez C., Luz M. Wintaco M., Diana P. Amador M., Germán A. González M., Mary L. Carabalí I., Mónica Ararat S., Nury N. Olaya G., Luz H. Patiño B., Alfredo J. Sanabria S.

el colapso circulatorio en los pacientes afectados (Roberts y Perner, 2014).

Otras investigaciones sugieren que se debe ofrecer asistencia a quien se encuentre en una urgente necesidad y si existe una posibilidad de salvar al paciente, aspecto fundamentado en la denominada regla de rescate (the rule of rescue) (Shah et al., 2015). El "uso compasivo" de un agente por fuera de un protocolo científico con el fin de beneficiar a un paciente individual con una condición grave, sin otras alternativas disponibles, justifica el empleo de vacunas o medicamentos en casos específicos (Joffe, 2014).

Es éticamente aceptable ofrecer una intervención no probada y asumir un riesgo mayor (el cual tendrá que ser explicado al paciente, quien puede otorgar o no su consentimiento según su umbral aceptable de riesgo), si se trata de una condición crítica y no existe un tratamiento específico disponible (Rid y Emanuel, 2014b). Se puede justificar el suministro de medicamentos no probados que puedan mejorar potencialmente la condición de los infectados y, a la vez, recopilar datos científicos que contribuyan en la comprensión y control de la enfermedad (Donovan, 2014).

La Declaración de Helsinki (2013) habilita el uso de intervenciones no probadas en la práctica clínica, desde condiciones específicas. En el artículo 37, explica:

Cuando en la atención de un enfermo las intervenciones probadas no exis- ten $\mathrm{u}$ otras intervenciones conocidas han resultado ineficaces, el médico, después de pedir consejo de experto, con el consentimiento informado del paciente o de un representante legal autorizado, puede permitirse usar intervenciones no comprobadas, si, a su juicio, ello da alguna esperanza de salvar la vida, restituir la salud o aliviar el sufrimiento. Tales intervenciones deben ser investigadas posteriormente a fin de evaluar su seguridad y eficacia. En todos los casos, esa información nueva debe ser registrada y, cuando sea oportuno, puesta a disposición del público. (Asociación Médica Mundial, 2013, s. p.)

El uso de sueros en fase preclínica, en el contexto de la epidemia por el vE, puede considerarse una terapia emergente, más que un tratamiento en investigación. La tecnología emergente (o el procedimiento innovador) busca ofrecer un beneficio a un paciente específico en un escenario donde no existen otras opciones terapéuticas. Se define como procedimiento innovador a "una intervención nueva y en evolución, cuyos efectos primarios y secundarios, seguridad, confiabilidad y complicaciones potenciales no son aún bien conocidos por la comunidad de practicantes" (Shaul, Langer, McKneally, 2008). La innovación "participa en la fase exploratoria que precede a la investigación formal" (Shaul, Langer, McKneally, 2008). Por el contrario, el estudio busca responder una o varias preguntas de investigación, y está soportado en el rigor de un protocolo experimental, la 
incorporación de voluntarios de investigación, el registro de datos, la posibilidad de ser replicado y la incorporación a la práctica clínica si aprueba todas las fases clínicas de investigación.

En el contexto de la emergencia de salud pública, si hay razones empíricas (reportes de caso, desempeño en biomodelos, estudios de farmacocinética y toxicidad, etc.) para afirmar que el beneficio de la aplicación del suero en fase preclínica para los pacientes supera los riesgos de su aplicación y ofrece beneficios potenciales en comparación con el cuidado estándar, es posible asumir un riesgo mayor al mínimo, según el principio de proporcionalidad (Simonsen, 2012), siempre que el paciente o sus representantes otorguen su permiso después de un adecuado proceso de consentimiento informado.

En general se puede afirmar que está justificado éticamente el uso de terapias que no han sido probadas aun en humanos durante esta grave epidemia, sin que esto signifique que cesen los esfuerzos por conducir investigaciones dentro de los lineamientos éticos y jurídicos existentes, siempre y cuando:

- La relación costo-beneficio sea favorable para el paciente.

- Se cuente con el consentimiento plenamente informado de los participantes.

- Se tomen las medidas conducentes a salvaguardar la confianza pública en la profesión médica (Griffiths, 2014; Shah et al., 2015).
Los investigadores que administren medicamentos no comprobados tienen la obligación moral de verificar, valorar y comunicar los resultados, con el propósito de establecer su perfil de eficacia y seguridad. Los pacientes o sus representantes deben comprender de forma transparente que se trata de un medicamento que no ha surtido las fases de experimentación clínica. Se debe además garantizar la confidencialidad, libertad y dignidad de las personas y las poblaciones, lo que implica la participación de investigadores locales y la comunidad en la toma de decisiones. Asimismo, es necesario contar con una aprobación interdisciplinaria e independiente.

\section{¿Quiénes debieron ser los primeros en recibir los medicamentos expe- rimentales, de los cuales había pocos suministros?}

Hay una serie de consideraciones éticas que generalmente se aplican para decidir quiénes deben recibir terapias o medicamentos que no pueden suministrarse masivamente. La discriminación positiva es una de ellas; por lo tanto, es importante beneficiar a grupos sociales en condición de vulnerabilidad por cuestiones económicas o políticas como minorías étnicas, mujeres o personas de escasos recursos que por lo general no tienen acceso al sistema de salud (Pérez y Faccini-Martínez, 2015).

Meltzer y Childress (2008) proponen seguir criterios de selección que permitan alcanzar equidad entre una gran 
Boris J. Pinto B., Ana I. Gómez C., Luz M. Wintaco M., Diana P. Amador M., Germán A. González M., Mary L. Carabalí I., Mónica Ararat S., Nury N. Olaya G., Luz H. Patiño B., Alfredo J. Sanabria S.

variedad de grupos sociales en el suministro de medicamentos. No obstante, las particularidades de la epidemia por el VE imponen diferentes dificultades en el suministro proporcional y equitativo de los pocos medicamentos disponibles (Gostin et al., 2014). En este escenario, puede justificarse un enfoque utilitarista.

Esta perspectiva parte de la idea de maximizar el beneficio para la mayor cantidad de actores en términos de costo-efectividad (Jecker, 2015). Los criterios para la aplicación del enfoque utilitarista incluyen la gravedad del paciente, la probabilidad de beneficio, la cantidad de recursos necesarios y el tiempo que se usó en la intervención. En vista de los pocos suministros (que limitan su uso masivo), se debe realizar una selección tomando en consideración parámetros clínicos, así como el estadio de la enfermedad en el que se ha observado una mejor respuesta del paciente frente al tratamiento, con el fin de identificar la población que potencialmente exhiba una mejor respuesta. Así, para el suministro de medicamentos se priorizarían aquellos enfermos que presenten mayores probabilidades de recuperación gracias a la intervención y que demanden una utilización más eficiente de recursos.

Desde esta perspectiva, los miembros de los equipos de salud deberían recibir atención prioritaria, por el mayor riesgo de exposición, el rol que desempeñan en el control de la epidemia y el principio de reciprocidad, tomando en consideración que muchos de estos trabajadores de la salud, provenientes de países occidentales, ofrecieron su trabajo voluntario en el contexto de la enfermedad por el vE (Jecker et al., 2015; Griffiths, 2014; Gostin et al. 2014). Los profesionales de la salud pueden entender más fácilmente, en un contexto experimental, la incertidumbre del riesgo al que se exponen, así como el concepto de placebo (en el caso de proyectos de investigación). También se incluirían a los familiares cuidadores de los pacientes que se exponen a un gran riesgo con escasas medidas de protección.

No obstante, no se incluyeron a trabajadores de la salud africanos que también estaban afectados por la enfermedad, en quienes también aplicaba el principio de reciprocidad, o a las personas de la comunidad que apoyaban el cuidado de los enfermos y la inhumación de los cadáveres (especialmente mujeres) sin entrenamiento médico, con escasas medidas de protección y expuestos a la infección; estos cumplían un papel fundamental en la contención de le epidemia (Kass, 2014). En este caso no se respetó el principio de equidad (distribución de los recursos según la necesidad), pues se justificó, desde el principio de reciprocidad, la asignación desigual por raza, etnicidad, estrato económico o género, sin una evidente razón científica que lo justificara (Council for International Organizations of Medical Sciences [cioms], 2002). El tratamiento experimental, cuyas existencias eran limitadas, debió ser provisto a quien más lo necesitara, mayor beneficio pudiera obtener y que se encontrara en alto riesgo de exposición. 
Algunos sugieren que la desconfianza pública frente a los investigadores occidentales, particularmente en África, podría justificar la priorización de personas que puedan otorgar de mejor manera un consentimiento informado; en este caso, a trabajadores de la salud provenientes de países occidentales, con el fin de que si el suero experimental resulta ser inefectivo y riesgoso, el tratamiento experimental administrado a personas africanas no terminaría por lesionar la frágil confianza pública en la ayuda médica occidental (Rid y Emanuel, 2014b). Quizá una propuesta intermedia pudo ser distribuir el ZMapp entre trabajadores de la salud, africanos y occidentales, tras un adecuado proceso de consentimiento informado y con medidas de soporte equivalentes, como las que recibieron los dos médicos norteamericanos en Estados Unidos.

El tratamiento experimental se pudo probar en personas que cumplieran con las características étnicas y sociodemográficas de la región, y que representaran a las comunidades que han convivido con el virus por varios años. Esta es una justificación importante si se piensa en avanzar en investigación sobre tratamientos efectivos basados en anticuerpos, que puedan ser extrapolables en estas regiones del África.

\section{El dinero que se invierta en esos fár- macos, iestaría mejor invertido en suministros para la cuarentena y edu- cación pública para ayudar a preve- nir la transmisión de la enfermedad?}

La enfermedad por el ve fue identificada hace 40 años, con brotes que hasta la epi- demia de 2014 habían sido controlados con base en prácticas adecuadas de higiene y educación (Nkoghé et al., 2004). En este momento, esta enfermedad es una prioridad de seguridad nacional para los Estados Unidos y Europa, no solo por el riesgo del cruce de fronteras en un mundo globalizado, sino por la posibilidad de que sea empleada como arma del bioterrorismo, lo cual explica el repentino interés por el desarrollo de productos efectivos en el corto plazo (Rid y Emanuel, 2014a). No obstante, el énfasis en el desarrollo de productos terapéuticos no debe desplazar la consideración sobre determinantes sociales, económicos y políticos para contener la expansión de la enfermedad.

Una de las principales causas de la expansión de la reciente epidemia por el ve en África Occidental es la precariedad de la infraestructura y dotación clínica en los países afectados (Komesaroff y Kerridge, 2014; Farmer, 2014). Por esta razón, se debe priorizar la destinación de recursos al mejoramiento de la infraestructura clínica (Shah et al., 2014; Rid y Emanuel, 2014b; Gericke, 2015). A esto se suma que el proceso de investigación y aprobación de medicamentos puede ser largo, complejo y de resultados inciertos, por lo que priorizar el suministro de elementos de cuarentena y educación pública (con el empleo de protocolos ya existentes) puede evitar muchas muertes de forma efectiva (Nkoghé et al., 2004; Pérez y Faccini-Martínez, 2015).

La inversión en la dotación clínica y el entrenamiento para los trabajadores de 
Boris J. Pinto B., Ana I. Gómez C., Luz M. Wintaco M., Diana P. Amador M., Germán A. González M., Mary L. Carabalí I., Mónica Ararat S., Nury N. Olaya G., Luz H. Patiño B., Alfredo J. Sanabria S.

la salud que exponen sus vidas en las zonas más críticas de la epidemia ha sido una necesidad ineludible (Boozary et al. 2014), dado que los países afectados por la epidemia tienen la menor tasa de personal médico (médicos y enfermeras) para atender las necesidades de la población (Donovan, 2014). Por ejemplo, Liberia tiene un médico por cada 100000 habitantes, comparado con 240 en Estados Unidos o 670 en Cuba (Farmer, 2014). Este tipo de mejoras pueden fortalecer el sistema de salud frente a futuras epidemias y contribuir al tratamiento actual de un gran número de enfermedades (Rid y Emanuel, 2014b).

Los cuidados sistemáticos, buenas prácticas de higiene y la rápida respuesta de los sistemas de salud para identificar la epidemia han sido los pilares para el control y mitigación de los brotes (Nkoghé et al., 2004; Pérez y Faccini-Martínez, 2015). Se afirma entonces que los resultados positivos del tratamiento en los ciudadanos americanos se generaron en gran medida por los cuidados intensivos, la rehidratación y suplementación oportuna de electrolitos (Lyon et al., 2014); asimismo, puede hablarse positivamente del caso de un paciente ugandés, reportado en Alemania (cuyos costos de tratamiento excedieron el millón de euros) tratado con éxito en un hospital en Frankfurt (Zacharowski, Brodt, Wolf, 2015).

Diversos trabajos señalan la importancia de identificar otras intervenciones potenciales para controlar estas epidemias, que se sumen a los tratamientos tradicionales de higiene y manejo de la enfermedad, como los sugeridos por Nkoghé et al. (2004), así como destinar recursos económicos a problemas de salud pública (como el epicentro de la epidemia), más que a la experimentación (Nkoghé et al., 2004; Sha et al., 2015; Rid y Emanuel 2014b; Pérez y Faccini-Martínez, 2015; Gericke, 2015; Griffiths, 2014).

Existen alternativas de control que no implican altos costos y podrían desarrollarse paralelamente a la investigación de los medicamentos, como son: tratar de evitar el contacto directo con pacientes infectados y con animales que puedan ser riesgo de contagio. En el caso de consumo, realizar una adecuada cocción de productos animales antes de consumirlos, y optimizar las condiciones de higiene (manejo de residuos contaminados y lavado de manos) (CDC, 2015b).

No obstante, persistir en la negativa ante el desarrollo de estrategias preventivas y terapéuticas para la enfermedad por el VE implicaría reforzar la brecha entre esfuerzos y recursos destinados a investigación y necesidades de salud pública (la brecha 10/90) (Pinto et al. 2014). Por 40 años y después de 25 brotes, se ha denunciado el nulo interés en el desarrollo de tratamientos efectivos para la enfermedad por el VE, así como para otras necesidades de salud que no revisten interés para el mercado global de medicamentos. No parece coherente rechazar esfuerzos de investigación cuando por fin se destinan recursos para su desarrollo.

En diciembre de 2014, el Gobierno americano firmó un contrato con la 
biofarmacéutica Mapp para acelerar el desarrollo de la droga ZMapp por US\$ 24,9 millones, con la posibilidad de extenderlo al doble: US\$ 42,3 millones (McCarthy, 2014). Las inversiones hechas por Estados Unidos en medicamentos para combatir la enfermedad por el VE representan menos del 4\% del presupuesto anual que invierten los países de la epidemia (Guinea, Liberia y Sierra Leona) en salud pública. Este es un coste marginal que, además de no ser asumido por los países de riesgo, puede constituir una opción conveniente de lucha contra futuros brotes.

En el caso de las vacunas, una vez que estos productos sean obtenidos, deben estar disponibles a costos asequibles para la población en riesgo (McCarthy, 2014). Este es el caso de las vacunas que se encuentran en fase I y fase II de investigación (una basada en adenovirus, otra en el virus de la estomatitis vesicular y otras vacunas recombinantes) (Instituto de Salud Global de Barcelona [Isglobal], 2016).

Los recursos se deben priorizar diferenciando entre aquellas intervenciones que son a largo plazo y genéricas del uso de medicamentos no probados en el corto plazo y a escala individual (Gericke, 2015). Si bien el desarrollo de fármacos es importante, la reducción de la incidencia y prevalencia se dio gracias a estrategias educativas y a la implementación de medidas de bioseguridad para limitar la transmisión. También se logró a través de la incorporación de estudios antropológicos que han profundizado la comprensión sobre las dinámicas de interacción ecológica entre humanos y murciélagos; la importancia del contacto entre los vivos y los muertos en el contexto de los ritos funerarios en estas comunidades como expresión de los lazos que mantienen el tejido social, los procesos de duelo y sus repercusiones epidemiológicas, así como la percepción de desconfianza de la comunidad ante las prácticas de cuarentena y las medidas de contención de los sistemas de salud pública. La indagación antropológica ha permitido una mejor colaboración de las comunidades en la adopción de prácticas seguras de enterramiento, al tiempo que se preserva la importancia de sus ritos funerarios (Marí Sáez et al., 2014).

El tratamiento con medicamentos experimentales contra el virus del Ébola, ¿debería ofrecerse exclusivamente en el contexto de ensayos clínicos controlados aleatorizados?, ¿o existen otras alternativas que permitan generar conocimiento sobre el perfil de seguridad y eficacia de tales medicamentos?

En un ensayo clínico controlado aleatorizado (ECA), los participantes son asignados por azar a uno $\mathrm{u}$ otro grupo y reciben diferentes intervenciones, que pueden ser un medicamento experimental, un medicamento convencional o un placebo. Los datos son analizados y comparados para responder las preguntas de la investigación (Presidential Commission for the Study of Bioethical Issues, 2015).

En este caso, un grupo recibiría una nueva intervención más el cuidado convencional 
Boris J. Pinto B., Ana I. Gómez C., Luz M. Wintaco M., Diana P. Amador M., Germán A. González M.,

Mary L. Carabalí I., Mónica Ararat S., Nury N. Olaya G., Luz H. Patiño B., Alfredo J. Sanabria S.

y otro grupo, cuidado convencional más placebo. Este tipo de diseño, si bien ofrece la mayor aproximación al método experimental y permite compilar resultados científicos de forma más rápida sobre la eficacia de un tratamiento específico (Lazcano-Ponce et al., 2004), brinda una serie de problemáticas éticas, como el mecanismo para definir quiénes son asignados a un grupo u otro y si es moralmente correcto negar a unas personas la posibilidad que podría salvar su vida, entre otras. Se configura como un conflicto entre eficacia y equidad (Kass, 2014).

La primera consideración que hay que tratar en este punto es la vulnerabilidad situacional y patogénica (Mackenzie et al., 2014) de las poblaciones en África subsahariana, en zonas marginales cercanas a selvas tropicales, donde las comunidades humanas se encuentran inmersas en la pobreza y carentes de un sistema sólido de salud pública (Pérez y Faccini-Martínez, 2015; Pourrut et al., 2005), en el contexto de gobiernos debilitados por guerras civiles y fragilidad institucional y con altos índices de mortalidad por enfermedades infecciosas y parasitarias.

Otros autores sugieren que, pese a que los ECA son considerados como la prueba de oro para evaluar el perfil de seguridad y eficacia de medicamentos, así como la obtención de la licencia aprobatoria de su uso, en el contexto de la enfermedad por el ve existen muchas consideraciones que hacen controversial su aplicación. Frente a la urgencia por salvar la vida, cada persona podría romper las consideraciones y recomendaciones suscritas al aplicarles el efecto placebo (Waldman y Nieburg, 2015).

Por otra parte, en este caso no se justifica la aleatorización con grupo placebo, pues no hay equiponderación (equipoise). La aleatorización con grupo placebo se justifica si hay genuina incertidumbre entre dos alternativas. En el caso de la enfermedad por el vE, el cuidado convencional ofrece muy poco beneficio y la mortalidad es excesivamente alta (alrededor del 70\%) (Adebamowo et al., 2014). No obstante, para algunos autores rechazar la aleatorización con grupo control implica asumir que los productos en investigación son eficaces y seguros, lo cual es precisamente lo que cabe investigar (Hayden, 2014).

De acuerdo con Caplan et al. (2015), dadas las múltiples limitaciones metodológicas y prácticas, se deben buscar alternativas de análisis rigurosas y consistentes desde el punto de vista metodológico. Una posibilidad es reemplazar en los ECA el placebo por "controles activos"; dado que no existe tratamiento avalado, se deben implementar otros que se usen como alternativas de atención de la sintomatología de la enfermedad (Cohen y Kupferschmidt, 2014; Caplan et al., 2015).

Otra posibilidad son los estudios con enfoques bayesianos en los cuales se puede superar la necesidad de emplear un grupo asignado a placebo (en el contexto de ECA dentro de los métodos frecuentistas), utilizando grupos de control compartido que permiten la 
incorporación de los medicamentos que demuestren efectividad al grupo de control con medidas de cuidado estándar (Cox et al., 2014).

Otro modelo sugerido es el denominado ensayo de diseño adaptativo, que consiste en asignar tratamientos diferenciales con la posibilidad de cambiar las condiciones del ensayo en la medida que se observa la evolución clínica (Coffey et al., 2012) (Kass, 2014).

Se pueden proponer otros diseños alternativos que también ofrecen validez estadística. De esta forma, se testean rápidamente aquellos tratamientos que ofrecen mayores beneficios y se descartan otros. Son similares a los estudios de fase II que son aceptados por las instancias de control (Adebamowo et al., 2014).

Se sugiere también el control histórico de datos de ensayos terapéuticos, el cual consiste en comparar los resultados de los individuos que reciben el medicamento dentro de un ensayo clínico con los de personas en situaciones similares que no hacen parte del protocolo (Presidential Commission for the Study of Bioethical Issues, 2015).

Teniendo en cuenta que existen las alternativas mencionadas, entre otras, para generar conocimiento sobre el perfil de seguridad y eficacia de los medicamentos experimentales, es importante que en el contexto de la epidemia por el vE se exploren los diferentes modelos que desde las normas éticas y morales eviten la segregación y exclusión de personas en estado de vulnerabilidad, manteniendo la integridad científica y estadística.

No obstante, el Instituto Nacional de Salud de Estados Unidos (NIH) ha proseguido con los estudios del medicamento en conjunto con el Gobierno de Liberia, y en estos ha planteado investigaciones clínicas de "casos y controles", lo que implica tratar solo a un grupo de la población voluntaria que acceda al estudio; de esta manera le da el beneficio del medicamento al grupo de casos solamente, donde los resultados se evaluarán en cuanto se presente la disminución de las muertes en los pacientes tratados. Esto genera un conflicto ético respecto a la posibilidad de recibir el tratamiento, en el contexto de los estudios de eficacia planteados de esta manera (Rid y Emanuel, 2014b).

\section{¿Qué consideraciones éticas deben tomarse en cuenta en el desarrollo de vacunas contra el virus del Ébola?}

Si bien existen otros métodos útiles para evitar el contagio de personas sanas (Shah et al., 2015), recomendaciones prácticas para el manejo de la enfermedad en pacientes infectados (Nkoghé et al., 2004), así como posibilidades de control de la epidemia desde su epicentro, evaluando el contexto social y los factores de propagación natural (Pérez y Faccini-Martínez, 2015), la vacuna es otra alternativa de prevención primaria.

En el contexto de urgencia por la enfermedad por el ve, se han planteado ocho ensayos clínicos para probar tratamientos contra la enfermedad del ve y once 
Boris J. Pinto B., Ana I. Gómez C., Luz M. Wintaco M., Diana P. Amador M., Germán A. González M., Mary L. Carabalí I., Mónica Ararat S., Nury N. Olaya G., Luz H. Patiño B., Alfredo J. Sanabria S.

ensayos clínicos para probar vacunas (oms, 2014c). En el desarrollo de una vacuna y en los estudios experimentales que lo soportan, se pueden aplicar diferentes principios éticos de la Declaración de Helsinki, las pautas éticas cıoms, el Informe Belmont y el Código de Núremberg. Emanuel, Wendler y Grady (2008) han propuesto algunos requisitos éticos generales para investigaciones clínicas que son compatibles con la mayoría de las directrices y acuerdos presentes en estos documentos.

- Valor social de la investigación.

- Validez científica del estudio.

- Selección equitativa de los participantes.

- Relación favorable entre riesgos y beneficios.

- Proceso de consentimiento informado.

- Revisión independiente del estudio.

- Respeto por los derechos de los participantes.

- Investigación participativa basada en la comunidad.

De estos principios enunciados es importante resaltar tres que se pueden considerar de alta importancia en el contexto de la epidemia por el vE: la selección equitativa de los participantes, el proceso de consentimiento informado y la asociación colaborativa para la investigación.

La selección equitativa de los participantes es clave, particularmente para los países del África Occidental donde la mayoría de la población vive en condición de vulnerabilidad, hay bajo cubrimiento en salud y en algunos casos poca confianza hacia el personal médico (Shah et al., 2015; Griffiths, 2014). Meltzery Childress (2008) señalan que los ensayos clínicos experimentales deben distribuir de forma equitativa los posibles efectos (positivos y negativos) de las intervenciones. Para ello recomiendan aplicar un principio de proporcionalidad social en la selección de pacientes, de tal manera que no exista un sesgo hacia sectores que típicamente han recibido beneficios en ensayos clínicos, como los hombres o grupos adinerados. Si existe una alta posibilidad de efectos negativos colaterales, no es recomendable sobre-representar en los ensayos a personas que por lo general han asumido esta carga en ensayos médicos, como minorías, grupos marginados, mujeres o presidiarios (Emanuel, 1999). De igual manera, se debe garantizar acceso equitativo a los beneficios identificados en los estudios (Folayan et al., 2016).

En este sentido, hay puntos interesantes en el desarrollo de vacunas contra el ve: los estudios en fase I se realizaron en Canadá, en Halifax, con voluntarios canadienses que respondieron masivamente a la convocatoria y que, recibieron un estipendio por su participación (alrededor de 1100 dólares por 11 visitas) (Taber, 2014); sin embargo, se consideró que su contribución no obedeció al monto de la compensación (en una ciudad con un pib per cápita de alrededor de 45700 dólares) (Morley y Macleod, 2015), sino al deseo solidario de contribuir en la contención de la epidemia en África Occidental.

Por otra parte, los datos preliminares de uno de los estudios en fase III para el 
desarrollo de una vacuna, realizado en Guinea, sugiere un buen perfil de eficacia y seguridad de la vacuna rVSV-ZEBOV (basada en el virus de la estomatitis vesicular), mediante un diseño aleatorizado de vacunación de conglomerados por anillos, en el cual se vacunan en diferentes momentos (día 0, día 21 tras la posible exposición), contactos de pacientes afectados por la enfermedad por ve. Este es un diseño que se adapta a las necesidades técnicas del estudio en cuanto a demostración de eficacia, efectividad, capacidad inmunogénica y seguridad de la vacuna, al tiempo que es aceptable desde un punto de vista ético, en preferencia a diseños aleatorizados con grupo placebo (Rid y Miller, 2016).

Es importante, no obstante, esperar con cautela los resultados finales de los estudios en curso y no ofrecer estos datos preliminares (que sugieren una efectividad del $100 \%$ tras la vacunación inmediata) como un incentivo para ejercer alguna forma de coerción a trabajadores de la salud, que representan la primera línea de defensa en el contexto de la epidemia (Folayan et al., 2016).

Con respecto al consentimiento informado, este proceso se fundamenta en el principio de respeto a la autonomía de los voluntarios de investigación y tiene como propósito promover la libertad de decisión de las personas. En el área de influencia de la epidemia existe una gran variedad de grupos étnicos con diferentes visiones y percepciones frente a la salud y la enfermedad, por lo que un consentimiento informado con enfoque diferencial representa un elemento fundamental en el desarrollo de los ensayos para el desarrollo de la vacuna contra el vE.

Por último, es necesario un enfoque participativo basado en la comunidad. La historia de la ética en investigación ha pasado por varias etapas: el paternalismo de los investigadores, el proteccionismo regulatorio, el acceso de los participantes a los beneficios de la investigación biomédica, hasta el paradigma que debiera regir la investigación contemporánea: investigación participativa basada en la comunidad (Emanuel, 2008, p. 224). La percepción de desconfianza está relacionada con experiencias negativas pasadas con compañías farmacéuticas occidentales y con recientes cuestionamientos que han surgido en el contexto de esta epidemia; por ejemplo, el trato médico diferencial a enfermos de países occidentales o la falta de interés en el desarrollo de una vacuna a pesar de las epidemias registradas en las últimas décadas (Donovan, 2014). Un diálogo público honesto debe promoverse entre investigadores, comunidades y comités de ética, como un elemento fundamental en el impulso de los esfuerzos de investigación en torno al desarrollo de vacunas contra la enfermedad por el ve (Folayan et al., 2016).

La investigación participativa basada en la comunidad puede contribuir a la definición de los estándares de prevención para los participantes en investigación, el fortalecimiento de los sistemas de salud (Folayan y Haire, 2015), una mejor difusión de los resultados de investigación, en la construcción de 
Boris J. Pinto B., Ana I. Gómez C., Luz M. Wintaco M., Diana P. Amador M., Germán A. González M.,

Mary L. Carabalí I., Mónica Ararat S., Nury N. Olaya G., Luz H. Patiño B., Alfredo J. Sanabria S.

confianza pública, en la prevención de la falacia terapéutica, en la generación de compromisos en cuanto a la construcción de capacidad investigativa de los países locales y en evitar que casos de investigación extractiva (como los del Trovan de Pfizer y el Tenofovir) se vuelvan a repetir en África (Folayan, Peterson y Kombe, 2015).

\section{¿Cómo deben colectarse, almacenarse y compartirse los materiales bioló- gicos para investigar sobre el virus del Ébola?}

La Comisión Presidencial para el Estudio de Dilemas Bioéticos en Estados Unidos considera que la creación de biobancos para investigar sobre el ve es un tema prioritario. Se requieren bioespecímenes tanto de personas que han sobrevivido a la infección, como de aquellos que se encuentran enfermos o han fallecido, los que solo están disponibles durante la epidemia. Para la recolección de estos diversos tipos de materiales biológicos se necesita prestar especial atención al consentimiento y a la protección de la privacidad (en especial debido al estigma asociado con la enfermedad), de tal forma que puedan ser empleados y compartidos para diferentes tipos de investigación, que incluyen la decodificación de los genomas de los diferentes tipos de virus, así como la identificación de secuencias genéticas en las personas asociadas con la predisposición o la protección natural contra la enfermedad (Presidential Commission for the Study of Bioethical Issues, 2015).
La oms estudia la posibilidad de crear un biobanco con las más de 100000 muestras de semen, sangre, orina y leche materna, entre otros materiales biológicos, de personas infectadas o sospechosas de estarlo que han sido colectados por diferentes agencias internacionales durante la epidemia y darles un buen uso. Uno de los mayores problemas que enfrenta esta iniciativa es la alta contagiosidad de estos bioespecímenes con el subsecuente riesgo para los investigadores o la sociedad. El almacenamiento y uso de estos requiere condiciones de alta seguridad, con una clasificación del ve en categoría A de sustancias infecciosas, lo cual exige un laboratorio de contención con nivel de bioseguridad tipo II, además de personal altamente calificado; esto, debido a las condiciones de los países de origen, es difícil de lograr.

Muchas de las muestras se encuentran actualmente en biobancos del Instituto de Medicina Tropical de Hamburgo en Alemania, el CDC de Atlanta en Estados Unidos y el Instituto Pasteur en Francia, aunque países como Canadá consideran que extraer estas muestras de África puede representar un riesgo mayor. Laexportación a países industrializados de materiales biológicos con datos genéticos requiere aprobación de los comités de ética de origen y de destino y protección de la titularidad sobre los especímenes biológicos. La OMS propone asociar estas muestras a través de un biobanco online, con uno de referencia ubicado en África Occidental, y determinar cuáles deben ser las líneas prioritarias de investigación debido a que estos recursos son finitos (Check, 2015). 
Se cuestiona si el consentimiento para la obtención, almacenamiento y uso de estos especímenes debe ser amplio o si es necesario reconsentir para usos específicos. Si bien el tratamiento de datos genéticos requiere consentimiento con fines específicos, en este caso se recomienda un consentimiento amplio con unas propuestas determinadas de opciones en investigación, dadas las dificultades para reubicar a los titulares de los materiales biológicos (por la alta tasa de mortalidad y las limitadas condiciones de la infraestructura de salud). En muchos casos se necesitará un consentimiento sustituto, debido a que se trata de menores de edad, personas con incapacidad de otorgar su consentimiento o vulnerables dada su condición clínica (Presidential Commission for the Study of Bioethical Issues, 2015).

Como alternativa se propone la anonimización de las muestras para facilitar el tratamiento de especímenes y datos clínicos. Sin embargo, esta medida no garantiza el anonimato debido a que el material puede proveer información única de cada individuo y de sus familiares biológicos (a través del análisis de secuencias de ADN no codificante, como en el caso de cotejos en medicina forense o pruebas de paternidad).

El uso de estos materiales por otros investigadores requiere la aprobación de un comité de ética en investigación. También se debe asegurar que los beneficios obtenidos del uso de estos materiales biológicos sean asequibles para las comunidades de donde provienen
(Presidential Commission for the Study of Bioethical Issues, 2015).

Finalmente, una de las mayores lecciones aprendidas de la epidemia por el ve es la necesidad de crear redes interdisciplinares que tengan un objetivo común y permitan compartir rápidamente los resultados de la investigación. Esto se hizo evidente cuando grupos internacionales de investigadores y del Instituto de Cambridge, Massachusetts, decodificaron el genoma del virus proveniente de pacientes de Guinea y Sierra Leona e inmediatamente publicaron la información en la base de datos pública GenBank. Esta forma de actuar en ciencia, que no es la más frecuente, se debe soportar en principios como los que en su momento se expresaron en el acuerdo de Bermuda de 1996, como el hecho de que, a pesar de hacer rápidamente públicos los datos, los centros mantienen el derecho a publicar posteriormente (Yozwiak et al., 2015).

\section{Conclusiones}

Las dimensiones catastróficas de la epidemia por el ve en África occidental han obligado a repensar los procesos de investigación en situaciones de brote epidémico. Tanto el miedo de que dicha amenaza cruzara las fronteras hasta Europa y América, como sentimientos de solidaridad y altruismo, han sido los motores de los cambios observados. Como afirma Angus Deaton, el progreso genera desigualdades que, tras ciertos impulsos históricos (muchas veces por necesidad, por miedo o por avaricia), se diseminan lentamente a la mayoría de las 
Boris J. Pinto B., Ana I. Gómez C., Luz M. Wintaco M., Diana P. Amador M., Germán A. González M., Mary L. Carabalí I., Mónica Ararat S., Nury N. Olaya G., Luz H. Patiño B., Alfredo J. Sanabria S.

personas. La disminución de otras formas de desigualdad (como la desigualdad política) es el punto crítico y necesario para acelerar el gradiente de igualdad en el acceso a los beneficios del progreso social y tecnocientífico (Deaton, 2015).

Dentro de las lecciones de la epidemia por ve podemos citar las siguientes: el uso de medicamentos no autorizados o experimentales puede ser ético en el contexto de una epidemia si la relación riesgo-beneficio es favorable para los pacientes, en ausencia de otras medidas terapéuticas efectivas, según el principio de la regla del rescate. Consideramos, no obstante, que se debió priorizar al equipo de salud, pero desde un concepto extendido de este, es decir, que incluyera no solo a los médicos, enfermeros, auxiliares, sino también a los servicios de apoyo al proceso de atención, como pueden ser las personas responsables del aseo y del transporte e inhumación de los cuerpos, dado que la protección de su integridad se convierte en la primera barrera de contención de la epidemia. Esto significaría un uso eficiente del recurso escaso desde una perspectiva utilitarista y de equidad.

Se debe aprovechar esta coyuntura en cuanto a la voluntad de financiación internacional para el desarrollo de medicamentos y vacunas contra el VE, al tiempo que es importante priorizar el fortalecimiento de los sistemas de salud locales en los países afectados por la epidemia. El trabajo de antropólogos en el contexto de la epidemia por ve fue uno de los puntos fundamentales, no solo en la caracterización del origen zoonótico de la epidemia, sino también en la contención de su extensión, a partir del diálogo respetuoso con las comunidades y el conocimiento de sus prácticas culturales en torno a la muerte. En cuanto al diseño de los ensayos clínicos, se pueden proponer modelos alternativos que generen buenos resultados respecto al conocimiento del perfil de eficacia y seguridad de las intervenciones terapéuticas y preventivas, mientras se protegen los derechos de los voluntarios de investigación. Finalmente, una de las mayores lecciones aprendidas de la epidemia por el ve es la necesidad de crear redes interdisciplinares que tengan un objetivo común y permitan la rápida diseminación de los resultados de investigación (como el uso compartido de bases de datos genéticos entre distintos grupos de investigación).

Por último, tras el paulatino control de la epidemia, es necesario un espacio de reflexión que permita concretar en principios y directrices lo aprendido, sin perder de vista que la prioridad debe ser la protección de la vida, la integridad, la dignidad, la libertad y la intimidad de pacientes y voluntarios de investigación, así como el fortalecimiento de las capacidades locales en atención sanitaria. No obstante, el mayor reto que seguirá enfrentando la humanidad es cómo extender la igualdad de derechos, de oportunidades y la equidad para que cada persona pueda desarrollar su potencial humano. La participación activa de las comunidades y los gobiernos locales en estos esfuerzos es uno de los principales desafíos. 


\section{Referencias}

Adebamowo, C., Bah-Sow, O., Binka, F., Bruzzone, R. et al. (2014). Randomised controlled trials for Ebola: practical and ethical issues. The Lancet, 384(9952), 1423-4.

Asociación Médica Mundial [amm]. (2013). Declaración Helsinki, Principios éticos para las investigaciones médicas en seres humanos. 64ª Asamblea General. Fortaleza, Brasil.

Bausch, D.G., Sprecher, A.G., Jeffs, B., Boumandouki, P. (2008). Treatment of Marburg and Ebola hemorrhagic fevers: a strategy for testing new drugs and vaccines under outbreak conditions. Antiviral Research, 78(1), 150-161.

Benatar, S. (2015). Explaining and responding to the Ebola epidemic. Philosophy, Ethics, and Humanities in Medicine, 10(5), 1-3.

Bogoch, I.I., Creatore, M.I., Cetron, M.S., Brownstein, J.S. et al. (2015). Assessment of the potential for international dissemination of Ebola virus via commercial air travel during the 2014 west African outbreak. Lancet, 3(9962), 29-35.

Boozary, A.S., Farmer, P.E. y Jha, A.K. (2014). The Ebola Outbreak, Fragile Health Systems, and Quality as a Cure. Journal of the American Medical Association, 312(18), 1859-1860.

Caplan, A.L., Plunkett, C. y Levin, B. (2015). Selecting the right tool for the job. The American Journal of Bioethics, 15(4), 4-10.

Centers for Disease Control and Prevention [CDC] (2015a). Ebola (Ebola Virus Disease). Recuperado el 12 de noviembre de 2015, de http://www.cdc.gov/vhf/ Ebola/transmission/index.html
Centers for Disease Control and Prevention [cdc] (2015b). Enfermedad del virus del Ebola. Prevención. Recuperado el 18 de noviembre de 2015, de http:// espanol.cdc.gov/vhf/ebola/prevention/ index.html

Check, E. (2015). Proposed Ebola biobank would strengthen African science. $\mathrm{Na}$ ture, 524(7564), 146-147.

Coffey, C.S., Levin, B., Clark. C., Timmerman, C. etal. (2012). Overview, hurdles, and future work in adaptive designs: Perspectives from an NIH funded workshop. Clinical Trials, 9(6), 671-680.

Cohen, J. y Kupferschmidt, K. (2014). Ebola vaccine trials raise ethical issues: Randomized studies may offer fastest answer. Science Magazine, 346(6207), 289-290.

Council for International Organizations of Medical Sciences [cioms] y World Health Organization [wHo] (2002). Pautas éticas internacionales para la investigación y experimentación biomédica en seres humanos de la Organización Mundial de la Salud. Ginebra, pauta número 12. Recuperado el 21 de marzo de 2016, de http://www. cioms.ch/publications/guidelines/pautas_eticas_internacionales.htm

Cox, E., Borio, L. y Temple R. (2014). Evaluating Ebola therapies. The case for RCTs. New England Journal of Medicine, 371(25), 2350-2351.

Deaton, A. (2015). El gran escape. Salud, riqueza y el origen de la desigualdad. Madrid: Fondo de Cultura Económica.

Donovan, G.K. (2014). Ebola, epidemics, and ethics. What we have learned? Philosophy, Ethics and Humanities in Medicine, 9(15), 2-4. 
Boris J. Pinto B., Ana I. Gómez C., Luz M. Wintaco M., Diana P. Amador M., Germán A. González M., Mary L. Carabalí I., Mónica Ararat S., Nury N. Olaya G., Luz H. Patiño B., Alfredo J. Sanabria S.

Emanuel, E. (1999). ¿Qué hace que la investigación clínica sea ética? Siete requisitos éticos. En A. Pellegrini Filho y R. Macklin (Eds.), Investigación en sujetos humanos: experiencia internacional (pp. 33-46). Santiago: Organización Panamericana de la Salud.

Emanuel, E.J., Wendler, D. y Grady, C. (2008). An Ethical Framework for Biomedical Research. En J. Emanuel, C. Grady, R. Crouch, R. y F. Lie (Eds.), The Oxford textbook of clinical research ethics. Oxford: Oxford University Press.

Farmer, P.E. (2014). Diary. London Review of Books, 36(20), 38-39. Recuperado el 18 de diciembre de 2015, de http://www. lrb.co.uk/v36/n20/paul-farmer/diary

Feldmann, H., Klenk, H.D. y Sanchez, A. (1993). Molecular biology and evolution of filoviruses. Archives Virology, 7, 81-100.

Frieden, T.R., Damon, I., Bell, B.P., Kenyon, T. y Nichol, S. (2014). Ebola 2014. New Challenges, New Global Response and Responsibility. New England Journal of Medicine, 371, 1177-1180.

Folayan, M.O. y Haire, B. (2015). Ethical considerations in the conduct of research on therapies for the prevention and treatment of Ebola virus disease in developing countries. Pan African Medical Journal, 22(Suppl 1), 8.

Folayan, M.O, Peterson, K. y Kombe, F. (2015). Ethics, emergencies and Ebola clinical trials: the role of governments and communities in offshored research. Pan African Medical Journal, 22(Supp 1), 10.

Folayan, M.O., Yakubu, A., Haire, B., Peterson, K. (2016). Ebola vaccine development plan: ethics, concerns and proposed measures. BMC Medical Ethics, 17(10).
Gehring, G., Rohrmann, K., Atenchong, N. y Mittler, E. (2014). The clinically approved drugs amiodarone, dronedarone and verapamil inhibit filovirus cell entry. Journal of Antimicrobial Chemotherapy, 69(8), 2123-31.

Gericke, C.A. (2015). Ebola and ethics: autopsy of a failure. BMJ, 350, h2105.

Gostin, L.O., Lucey, D. y Phelan, A. (2014). The Ebola Epidemic: A Global Health Emergency. JAMA, 312(11), 1095-1096.

Griffiths, P.D. (2014). Ebola and ethics. Reviews in Medical Virology, 24(6), 363-364.

Hayden, E.C. (2014). Ethical dilemma for Ebola drug trials. Nature, 515(7526), 177-178.

Instituto de Salud Global de Barcelona [ISGlobal]. (2016). Ébola: dos años y 11300 muertes después. Recuperado el 17 de marzo de 2016, de http://www. isglobal.org/Ebola

Jecker, N.S., Dudzinski, D.M., Diekema, D.S. y Tonelli, M. (2015). Ebola virus disease. Ethics and emergency medical response policy. Chest, 148(3), 794-800.

Joffe, S. (2014). Evaluating novel therapies during the Ebola Epidemic. JAMA, 312(13), 1299-1300.

Kass, N. (2014). Ebola, Ethics, and Public Health: What Next? Annals of Internal Medicine, 161(10), 744-745.

Kilgore, P.E., Grabenstein, J.D., Salim, A.M., Rybak, M. (2015). Treatment of Ebola virus disease. Pharmacotherapy, 35(1), 43-53.

Komesaroff, P. y Kerridge. I. (2014). Ebola, Ethics, and the question of culture. Journal of Bioethical Inquiry, 11(4), 413-414. 
Kroll, D. (2014). Ebola 'Secret Serum': Small Biopharma, the army and Big Tobacco. Forbes-Pharma and Healthcare. Recuperado el 3 de marzo de 2016, de http://www. forbes.com/sites/davidkroll/2014/08/05/ Ebola-secret-serum-small-biopharmathe-army-and-big-tobacco/

Lazcano-Ponce, E., Salazar-Martínez, E., Gutiérrez-Castrellón, P., Angeles-Llerenas, A. et al. (2004). Ensayos clínicos aleatorizados: variantes, métodos de aleatorización, análisis, consideraciones éticas y regulación. Salud Pública de México, 46(6), 559-584.

Lyon, G.M., Mehta, A.K., Varkey, J.B., Brantly, K., et al. (2014). Clinical Care of Two Patients with Ebola Virus Disease in the United States. New England Journal of Medicine, 371(25), 2402-2409.

Mackenzie, C., Rogers, W. y Dodds, S. (2014). Vulnerability. New Essays in Ethics and Feminist Philosophy. New York: Oxford University Press.

Mapp Biopharmaceutical. (2014). ZMapp information sheet. Recuperado el 3 de marzo de 2016, de http://www.defyrus. com/images/ZMapp-information-sheet. pdf

Marí Sáez, A., Kelly, A. y Brown, H. (2014). Apuntes sobre el paciente cero: antropología en los tiempos del Ébola. La marea.com. Recuperado el 21 de marzo de 2016, de http://www.lamarea.com/2014/10/09/apuntes-sobre-el-caso-paciente-cero-antropologia-en-los-tiempos-del-ebola/

Marí Sáez, A., Weiss, S., Nowak, K, et al. (2015). Investigating the zoonotic origin of the West African Ebola epidemic. ЕмBO Molecular Medicine, 7, 17-23.
Mathers, C.D., Boerma, T. y Ma Fat, D. (2009). Global and regional causes of death. British Medical Bulletin, 92, 7-32.

Mathers, C.D. y Loncar, D. (2006). Projections of Global Mortality and Burden of Disease from 2002 to 2030. PLoS Medicine, 3(11), e442.

McCarthy, M. (2014). US signs contract with ZMapp maker to accelerate development of the Ebola drug. BMJ, 349, g5488.

Mehta, A.K., Varkey, J.B., Brantly, K., Pérez, C.E. y Faccini-Martínez, A.A. (2015). Epidemia de Ebola-201: enfocar los esfuerzos en el control desde su epicentro. Revista Panamericana de Infectología, 17(1), 5-6.

Meltzer, L. y Childress, J. (2008). What is a fair participant selection? (capítulo 35) En E. J. Emanuel, C. Grady, R. Crouch et al. (Eds.), The Oxford textbook of clinical research ethics (pp. 377-383). Oxford: Oxford University Press.

Mickleburgh, S., Waylen, K. y Racey, P. (2009). Bats as bushmeat: a global review. Oryx, 43, 217-234.

Morley, F. y Macleod, R. (2015). The Halifax Index 2015. An economic gut check with insights for action. (2015). Halifax partnership. Connect, collaborate, prosper. Recuperado el 21 de marzo de 2016, de http://www.halifaxpartnership. com/site/media/Parent/8x10_HalifaxIndex2015_June18_Web.pdf

Murray, C.J.L., Rosenfeld, L.C., Lim, S.S., Andrews, K.G. et al. (2012). Global malaria mortality between 1980 and 2010: a systematic analysis. The Lancet, 379(9814), 413-431.

Nkoghé, D., Formenty, P., Nnégué, S., Toung Mvé, M. et al. (2004). Recommandations 
Boris J. Pinto B., Ana I. Gómez C., Luz M. Wintaco M., Diana P. Amador M., Germán A. González M.,

Mary L. Carabalí I., Mónica Ararat S., Nury N. Olaya G., Luz H. Patiño B., Alfredo J. Sanabria S.

pratiques pour la prise en charge sur le terrain des patients infectés par le virus Ebola. Médecine Tropicale, 64(2), 199-204.

Organización Mundial de la Salud [омs] (2014a). Consideraciones éticas sobre el uso de intervenciones no registradas en la enfermedad por el virus del Ébola. Recuperado el 12 de octubre de 2015, de http://www.who.int/mediacentre/ news/statements/2014/Ebola-ethical-review-summary/es/

Organización Mundial de la Salud [oms] (2014b). Statement on the WHO Consultation on potential Ebola therapies and vaccines. Recuperado el 21 de marzo de 2016, de http://www.who. int/mediacentre/news/statements/2014/ Ebola-therapies-consultation/en/

Organización Mundial de la Salud [oms]. (2015a). Ebola Virus Disease. Recuperado el 26 de febrero de 2016, de http:// www.who.int/mediacentre/factsheets/ fs103/en/

Organización Mundial de la Salud [oms] (2015b). Ebola Situation Reports. Recuperado el 15 de diciembre de 2015, de http://apps.who.int/Ebola/Ebola-situation-reports

Organización Mundial de la Salud [oms] (2015c). Ebola data and satistics. Key indicators relevant to Ebola outbreak. Recuperado el 12 de noviembre de 2015, de http://apps.who.int/gho/data/ node. Ebola.keyind?lang=en

Pinto, B.J., Mojica, M.C., Gulfo, R., Endo, J.H., y Idárraga, J. (2014). Bioética y la brecha 10/90: fallos, desafíos y oportunidades. Revista Redbioética, 2(10), 81-93.
Presidential Commission for the Study of Bioethical Issues. (2015). Ethics and Ebola Public Health Planning and Response. Recuperado el 16 de diciembre de 2015, de http://bioethics.gov/sites/ default/files/Ethics-and-Ebola_PCSBI_508.pdf

Programa Conjunto de las Naciones Unidas sobre el VIH/SIDA [ONUSIDA] (2015) Diapositivas clave sobre epidemiología, julio 2015. Recuperado el 19 de julio de 2015, de http://www.unaids.org/sites/ default/files/media_asset/20150714_ epi_core_es.pdf

Pourrut, X., Kumulungui, B., Wittmann, T., Moussavou, G. et al. (2005). The natural history of Ebola virus in Africa. Microbes and Infection, 7(7-8), 1005-1014.

Qiu, X., Wong, G., Audet, J., Bello, A. et al. (2014). Reversion of advanced Ebola virus disease in nonhuman primates with ZMapp. Nature, 514(7520), 47-53.

Rid, A y Emanuel, E. (2014a). Why Should High-Income Countries Help Combat Ebola? JAMA, 312(13), 1297-1298.

Rid, A. y Emanuel, E. (2014b). Ethical considerations of experimental interventions in the Ebola outbreak. The Lancet, 384(9957), 1896-1899.

Rid, A. y Miller, F. (2016). Ethical Rationale for the Ebola "Ring Vaccination" Trial Design. American Journal of Public Health, 106(3), 432-5.

Roberts, I. y Perner, A. (2014). Ebola virus disease: clinical care and patient-centred research. The Lancet, 384, 2001-2002.

Shah, S., Wendler, D. y Danis, M. (2015). Examining the ethics of clinical use of unproven interventions outside of 
clinical trials during the Ebola epidemic. The American Journal of Bioethics, 15(4), 11-16.

Shaul, R.Z., Langer, J.C., McKneally, M.F. (2008). Innovación en cuidados médicos: ejemplos de cirugía. En A. Singer Peter y A. Viens (Eds.), The Cambridge Textbook of Bioethics. New York: Cambridge University Press.

Simonsen, S. (2012). Acceptable Risk in Biomedical Research. European Perspectives. Netherlands: Springer Science+Business Media.

Smith, D.I.H. (1978). Ebola haemorrhagic fever in Sudan, 1976. Bulletin of the World Health Organization, 56(2), 247-270

Taber, J. (2014). Hundreds of Nova Scotians volunteer for Ebola vaccine trial. The Globe and Mail. Recuperado el 21 de marzo de 2016, de http://www.theglobeandmail.com/life/health-and-fitness/ health/hundreds-of-nova-scotians-volunteer-for-Ebola-vaccine-trial/article21712654

United Nations Development Group [UNDG], Western and Central Africa. (2015).
Socio-Economic Impact of Ebola Virus Disease in West African Countries. A call for national and regional containment, recovery and prevention. United Nations Development Group. Recuperado el 16 de noviembre de 2015, de https://undg. org/main/undg_document/socio-economic-impact-of-ebola-virus-disease-in-west-african-countries/

Waldman, R. y Nieburg, P. (2015). Thoughts on Alternative Designs for Clinical Trials for Ebola Treatment Research. The American Journal of Bioethics, 15(4), 38-40.

Yozwiak, N.L., Schaffner S.F. y Sabeti, P.C. (2015). Make outbreak research open access. Nature, 518, 477-479.

World Health Organization Ebola Response Team[who-ert] (2014). Ebola Virus Disease in West Africa-the First 9 Months of the Epidemic and Forward Projections. New England Journal of Medicine, 371, 1481-1495. (2015). Medical treatment of an Ebola-infected doctor-ethics over costs? The Lancet, 385(9969), 685. 